\title{
Les hémocytes des Crustacés
}

\author{
par G. BAUCHAU \\ Laboratoire de Biologie animale, Faculté des Sciences, 5000 Namur, Belgique
}

Deux types cellulaires se distinguent aisément dans l'hémolymphe des crustacés : les cellules hyalines (6 à $8 \mu$ ) possédant un grand noyau sphérique central mais pas ou peu de granules dans le cytoplasme et les granulocytes $(13 \mu)$ au noyau souvent bilobé et au cytoplasme bourré de granules de forme et de taille variables $(0,3$ à 2,6 $\mu$ ). Entre ces deux types existe une série de cellules intermédiaires, plus ou moins riches en granules, de telle sorte qu'une différenciation progressive des cellules hyalines en cellules granuleuses semble constituer l'hypothèse la plus vraisemblable (Bauchau et De Brouwer, 1972). Une culture des hémocytes pourrait fournir à cet égard une preuve décisive. Nous n'avons pas observé de mitoses dans les cellules circulantes, mais certains granules contiennent une formation paracristalline rappelant une structure analogue présente dans les éosinophiles des Vertébrés supérieurs, arrivés au terme de leur maturation.

Le nombre des hémocytes varie en fonction du cycle de mue et d'intermue. Une augmentation accompagne la mise en route de la préparation de l'exuviation. De même, l'activité des gonades va de pair avec une multiplication des hémocytes de l'hémolymphe (Bauchau et Plaquet, 1973). Ces cellules interviennent également lors des processus de cicatrisation, en particulier après autotomie d'un péréiopode ; elles jouent aussi un rôle fondamental dans la gélification du plasma, plus ou moins complète selon l'espèce de crustacé étudié (Bauchau et De Brouwer, 1974).

De nombreux tests cytochimiques de coloration et de digestion enzymatique (pronase, pepsine, trypsine, alpha-amylase) indiquent clairement que les granules sont riches en mucosubstances neutres; ces éléments sont utilisés lors des réactions physiologiques où interviennent les hémocytes (Bauchau et al., 1975).

Les hémocytes sont capables de phagocytose (particules de carmin, de noir animal, levures, etc.), les granulocytes semblent plus actifs à cet égard que les autres hémocytes. D'autre part, le contact de corps étrangers avec l'hémolymphe (fils de cellulose, de coton, etc.) déclenche une réaction d'adhérence; ces matériaux se voient enrobés dans un manchon cellulaire, ce qui assure leur isolement. L'examen microscopique de ces capsules est en cours. 
Une analogie structurale et fonctionnelle se manifeste ainsi entre certains hémocytes au moins des crustacés, des insectes, des mollusques, de la Limule et des Onychophores. Cette constatation souligne l'intérêt d'une étude comparée des cellules sanguines appartenant à différents embranchements d'Invertébrés.

\section{Bibliographie}

BAuchau (A.-G.) et DE Brouwer (M.-B.), 1972. - Ultrastructure des hémocytes d'Eriocheir sinensis, crustacé décapode brachyoure. J. micr., 15, 171-179.

Bauchau (A.-G.) et Plaquet (J.-C.), 1973. - Variation du nombre des hémocytes chez les crustacés brachyoures. Crustaceana, 24, 215-223.

Bauchau (A.-G.) et DE Brouwer (M.-B.), 1974. - Etude ultrastructurale de la coagulation de l'hémolymphe chez les Crustacés. J. Micr., 19, 37-46.

Bauchau (A.-G.) De Brouwer (M.-B.), Passelecc-Gérin (E.) et Mengeot (J.-C.), $1975 .-$ Etude cytochimique des hémocytes des Crustacés décapodes brachyoures. Histochemistry, 45, 101-113. 